\title{
Alopecia neoplastica: An uncommon presentation of metastatic breast carcinoma
}

\section{Felipe Ladeira de Oliveira ${ }^{*}$, Luisa Kelmer Cortês de Barros Silveira ${ }^{2}$, Thais Schiavo Moraes ${ }^{3}$, Fernanda Helena Craide ${ }^{4}$, Maria Cristina Mejia Briceño ${ }^{5}$, Bernard Kawa Kac ${ }^{6}$}

\author{
${ }^{1}$ Department of Dermatology, Instituto Pós Graduação Izamar Millidiu da Silva, Rio de Janeiro, Brazil \\ ${ }^{2}$ Department of Dermatology, Instituto Nacional de Câncer, Hospital do Câncer I, Rio de Janeiro, Brazil \\ ${ }^{3}$ Department of Dermatology, Hospital Universitário Clementino Fraga Filho, Universidade Federal do Rio de Janeiro, Rio de Janeiro, Brazil \\ ${ }^{4}$ Department of Dermatology, Policlínica Geral do Rio de Janeiro, Rio de Janeiro, Brazil \\ ${ }^{5}$ Department of Dermatology, Hospital Mario Kroeff, Rio de Janeiro, Brazil \\ ${ }^{6}$ Diagnostika Lab, Rio de Janeiro, Brazil
}

\begin{abstract}
Cutaneous metastasis may correspond to the initial clinical presentation of hidden internal malignancies. In patients presenting said neoplasia, clinical manifestations of breast cancer reaches $23.9 \%$. Considering that neoplastic alopecia appears as an unusual pattern of the said metastasis, this report describes a case of such uncommon neoplastic alopecia which presents itself as a cutaneous metastasis of rapid progression in a patient with prior breast cancer history. We present a 47-year-old female patient reporting lesions at the scalp, and who was asymptomatic with a one-year evolution. The patient reported prior breast cancer history and presence of lung metastasis, and was undergoing chemotherapy at the time of consultation. A dermatological evaluation showed only a nodular lesion with erythematous surface and a diameter measuring about $4 \mathrm{~cm}$, firm in consistency, and immovable. She was routed to the Department of Dermatological Surgery, and the results from histopathology were consistent with a diagnosis of metastatic breast adenocarcinoma. Neoplastic alopecia appears as an unusual form of cutaneous metastasis which is predominantly described in association with breast cancer. The lesion's clinical features play a crucial role at the differential diagnosis, as the presence of erythema could distinguish neoplastic alopecia from alopecia areata. The existence of cutaneous metastasis leads to unfavorable outcomes. As a conclusion, cutaneous evaluation of patients is essential for treating visceral metastases, since the forms of cutaneous metastasis are diverse and can also affect the scalp.
\end{abstract}

Keywords: alopecia; breast cancer; metastasis; skin cancer

Citation: de Oliveira FL, de Barros Silveira LKC, Moraes TS, Craide FH, Briceño MCM, et al. Alopecia neoplastica: An uncommon presentation of metastatic breast carcinoma. J Surg Dermatol 2021; 6(1): 77; http://dx.doi.org/10.18282/jsd.v6.i1.77.

*Correspondence to: Felipe Ladeira de Oliveira, Rua Conselheiro Autran 35 apt 805, Vila Isabel, Rio de Janeiro, RJ, Brazil; oliveiraflmed@gmail.com

Received: $19^{\text {th }}$ August 2020; Accepted: $20^{\text {th }}$ November 2020; Published Online: $28^{\text {th }}$ November 2020

\section{Introduction}

Cutaneous metastasis may correspond to the initial clinical presentation of hidden internal malignancies but may also indicate a neoplasia relapse in previously stabilized oncological patients ${ }^{[1]}$. Such condition is seen in $0.7 \%$ to $10.4 \%$ of cancer patients, and thus is relatively unusual ${ }^{[2]}$. The mechanism of cutaneous involvement in the process

Copyright (C) 2021 de Oliveira FL, et al. This is an Open Access article distributed under the terms of the Creative Commons Attribu-tionNonCommercial 4.0 International License (http://creativecommons.org/licenses/by-nc/4.0/), permitting all non-commercial use, distribution, and reproduction in any medium, provided the original work is properly cited. 
of metastasis lies in the following dissemination pathways: hematogenic, lymphatic, direct invasion of the adjacent tissue, and iatrogenic — of which, the first two occupy a prominent position in the aforesaid mechanism $^{[3]}$. It is still worthy to mention that a study suggests different outcomes for cutaneous metastasis related to internal malignancies arising from different organs and that, among such conditions, breast cancer presents the best outcome ${ }^{[4]}$.

In this context, the incidence of cutaneous presentations of breast cancer in patients showing this kind of neoplasia reaches $23.9 \%{ }^{[2]}$. Among the several clinical cutaneous presentations of metastatic breast cancer, neoplastic alopecia appears as an unusual pattern of this metastasis, where neoplastic cells invade the dermis of the scalp and cause either direct or indirect damages to the pilosebaceous unit ${ }^{[5]}$.

Triggered by a distinct physiopathological mechanism that has not been completely clarified, neoplastic alopecia seems to have a multifactor origin ${ }^{[5]}$. Therefore, this report describes a case of such uncommon neoplastic alopecia which presents as a cutaneous metastasis of rapid progression in a patient with prior breast cancer history.

\section{Case report}

The patient is a 47-year-old female who was evaluated with a history of asymptomatic nodular lesion at the scalp, with a one-year evolution. She presented a prior breast cancer history in 2010 showing newly diagnosed lung metastasis, and was undergoing chemotherapy at the time of consultation.

Dermatological evaluation showed a nodular lesion (Figure 1) with erythematous surface and a diameter measuring about $4 \mathrm{~cm}$, firm in consistency and immovable, with evidence of surface telangiectasia, well-defined borders, and some superficial crusted lesions at the right frontoparietal region of the scalp but without discharge and fistula formation. The patient was routed to the Department of Dermatological Surgery and, due to a clinical suspicion of cutaneous metastasis, the above-described lesion was submitted for a biopsy.

The results from histopathology evidenced a neoplasia of the epithelial line, with atypical cells that form a lobular structure of isolated strings (Figures 2, 3, and 4), that metastatic breast adenocarcinoma was diagnosed. Currently, the patient still undergoes chemotherapy and is under follow-up for evaluation of the lesion.

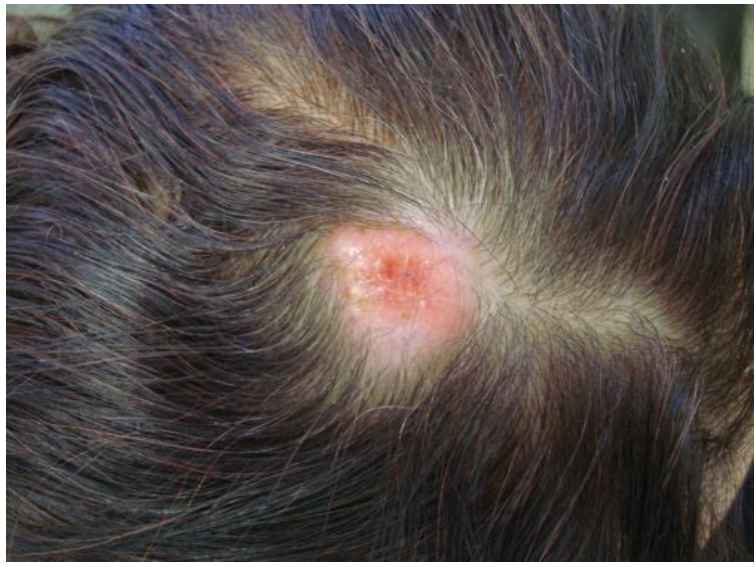

Figure 1. Nodular lesion showing superficial erythema and telangiectasias

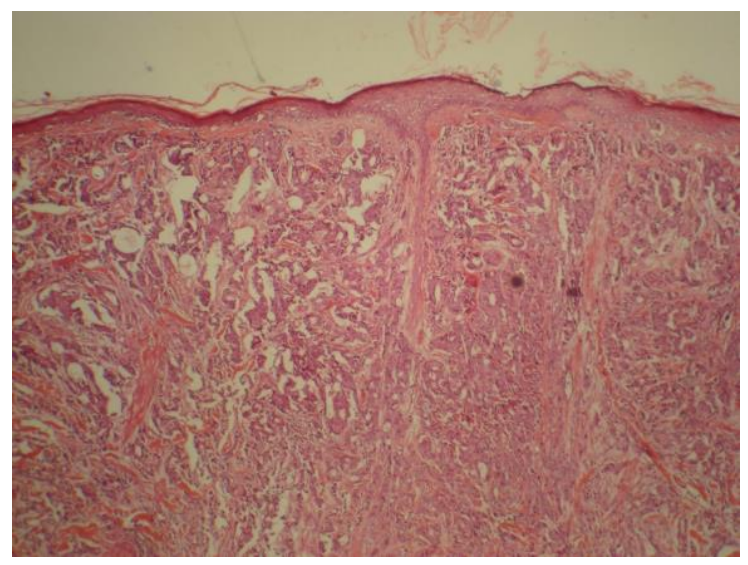

Figure 2. Cohesive and small cells forming cords or acinar outlines that invade the stroma [Hematoxylin and Eosin stain (H\&E) 100X]

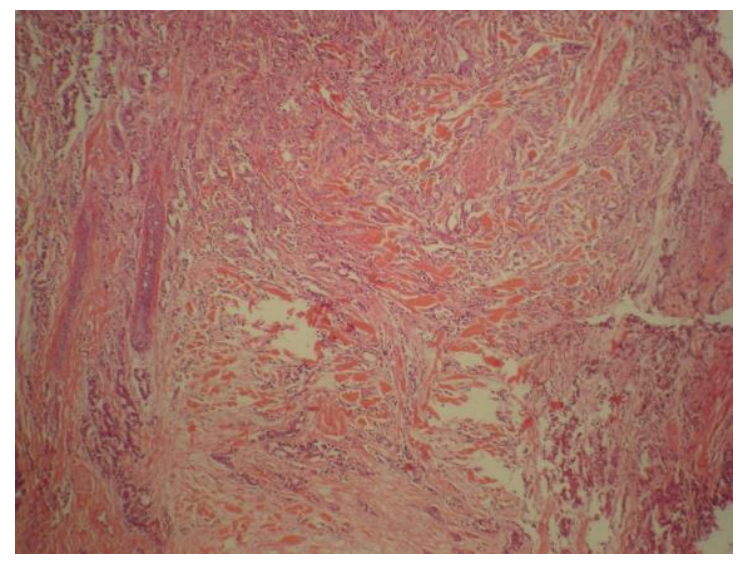

Figure 3. The cells display round to notched ovoid nuclei (H\&E 100X) 


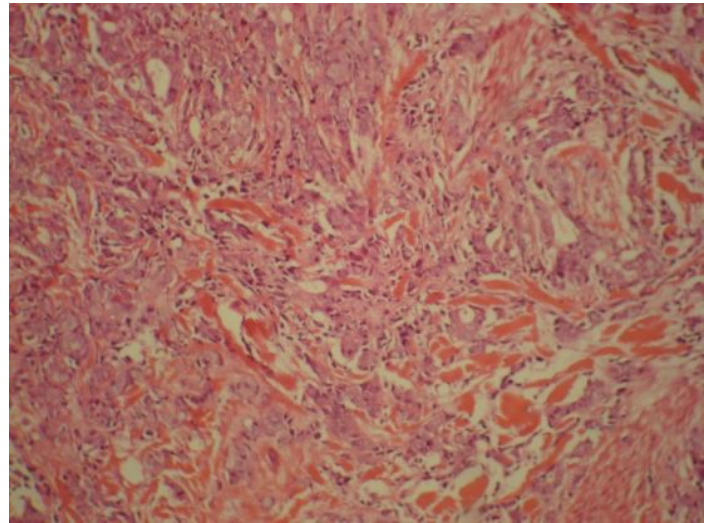

Figure 4. Interspersed thin collagen strands (H\&E 400X)

\section{Discussion}

Neoplastic alopecia is an unusual form of cutaneous metastasis, defined by localized hair loss caused by primary malignant neoplasia that progresses towards the scalp due to metastasis ${ }^{[2]}$. Only 25 cases have been described in literature so far, among which 21 are associated with breast cancer, thus representing $84 \%$ of these cases ${ }^{[5]}$.

Nevertheless, there are other clinical forms of cutaneous metastasis related to breast cancer, and the most frequent lesions are nodules, papules ${ }^{[6]}$, and tumoral lesions ${ }^{[7]}$, with the inflammatory form being described by a recent study as the most common ${ }^{[6]}$. Other forms, such as telangiectasias, zosteriform lesions, Paget-like metastasis, and subungual metastasis are also referred to as unusual events ${ }^{[2]}$.

It is worthy to point out the discrepancies between the clinical forms of unusual neoplastic alopecia, as several lesions showing different morphologic and quantitative patterns have already been described. Moreover, their sizes also present considerable variations, which may reach up to $20 \mathrm{~cm}^{[2,5]}$. It is still possible to verify cases of alopecia totalis due to breast cancer metastasis ${ }^{[8]}$. Such wide range of clinical forms are partially explained by the natural progression of metastatic conditions - at the beginning of its evolution, the area affected by alopecia is erythematous and well-marked ${ }^{[9]}$, as observed in the only lesion on the scalp of the patient. The lesion's clinical features also play a crucial role during differential diagnosis, as described by Cohen et $a l^{[9]}$. Given that the presence of erythema could distinguish neoplastic alopecia from alopecia areata, this clinical finding was seen in $41 \%$ of patients presenting neoplastic alopecia $^{[9]}$.

The most common histopathological findings are either single or multiple nodules at the dermis and subcutaneous tissues, which are constituted by tumor cell aggregates surrounded by fibrotic tissue ${ }^{[6]}$. It is possible to observe telangiectasia at the dermis above the tumoral area and even an enlargement of lymph vessels at areas of cutaneous metastasis and intralymphatic tumor emboli $^{[6]}$. It may be pointed out that adenocarcinoma is the cancer histological subtype that most often presents metastasis towards skin ${ }^{[10]}$.

As to outcomes, the presence of cutaneous metastasis lead to an unfavorable progression ${ }^{[10]}$. Nevertheless, some studies suggested different survival periods in cases of cutaneous metastasis originating from neoplasia in different organs, given that patients affected by breast cancer cutaneous metastasis present a relatively better outcome ${ }^{[3]}$. This fact was demonstrated by Schoenlaub et al., who described a longer survival period in patients affected by breast cancer cutaneous metastasis, in comparison to patients showing lung cancer and other kinds of neoplasia ${ }^{[4]}$.

The choice of chemotherapy for the referred patient was based on two relevant conditions presented: cutaneous metastasis of rapid progression ${ }^{[2]}$ and concomitant lung involvement.

\section{Conclusion}

It is essential that patients treated for visceral metastases undergo skin evaluation since the forms of cutaneous metastasis are diverse and can also affect the scalp.

\section{Conflict of interest}

The authors declare no potential conflict of interest with respect to the research, authorship, and/or publication of this article.

\section{References}

1. Hussein MRA. Skin metastasis: A pathologist's perspective. J Cutan Pathol 2010; 37(9): e1-e20. doi: 10.1111/j. 1600-0560.2009.01469.x.

2. De Giorgi V, Grazzini M, Alfaioli B, Savarese I, Corciova SA, et al. Cutaneous manifestations of breast carcinoma. Dermatol Ther 2010; 23(6): 581-589. doi: 10.1111/j. 1529-8019.2010.01365.x.

3. Hu SCS, Chen GS, Lu YW, Wu CS, Lan CC. Cutaneous metastases from different internal malignancies: A clinical and prognostic appraisal. J Eur Acad Dermatol Venereol 2008; 22(6): 735-740. doi: 10.1111/j.1468-3083 2008.02590.x.

4. Schoenlaub P, Sarraux A, Grosshans E, Heid E, Cribier B. (French) [Survival after cutaneous metastasis: A study of 200 cases]. Ann Dermatol Venereol 2001; 128(12): 13101315.

5. Conner KB, Cohen PR. Cutaneous metastasis of bre- 
ast carcinoma presenting as alopecia neoplastica. South Med J 2009; 102(4): 385-389. doi: 10.1097/SMJ. 0b013e31819b0c26.

6. Yun SJ, Park HY, Lee JS, Park MH, Lee JB, et al. Clinicopathological correlation of cutaneous metastatic breast carcinoma using lymphatic and vascular markers: Lymphatics are mainly involved in cutaneous metastasis. Clin Exp Dermatol 2012; 37(7): 744-748. doi: 10.1111/j. 1365-2230.2011.04306.x.

7. AbdullGaffar B, Almualla A, Al-Marzooqi F. Post-mastectomy breast rash. Int J Dermatol 2010; 49(8):
855-857. doi: 10.1111/j.1365-4632.2009.04453.x.

8. Martin J, Ross JB. Alopecia totalis as a presentation of cutaneous metastasis (alopecia neoplastica). Int $\mathbf{J}$ Dermatol 1983; 22(8): 487-489. doi: 10.1111/j.13654362.1983.tb02181.x.

9. Cohen I, Levy I, Schreiber H. Alopecia neoplastica due to breast carcinoma. Arch Dermatol 1961; 84(3): 490-492. doi: 10.1001/archderm.1961.01580150136023.

10. Rondina A, Kohen LL, Scherschun L, Kerr H. A woman with focal alopecia. J Am Acad Dermatol 2010; 63(3): 545-547. doi: 10.1016/j.jaad.2009.08.014. 